\title{
Seismic Event Location: Nonlinear Inversion Using a Neighbourhood Algorithm
}

\author{
M. S. SAmbridge ${ }^{1}$ and B. L. N. KennetT ${ }^{1}$
}

\begin{abstract}
A recently developed direct search method for inversion, known as a neighbourhood algorithm (NA), is applied to the hypocentre location problem. Like some previous methods the algorithm uses randomised, or stochastic, sampling of a four-dimensional hypocentral parameter space, to search for solutions with acceptable data fit. Considerable flexibility is allowed in the choice of misfit measure.

At each stage the hypocentral parameter space is partitioned into a series of convex polygons called Voronoi cells. Each cell surrounds a previously generated hypocentre for which the fit to the data has been determined. As the algorithm proceeds new hypocentres are randomly generated in the neighbourhood of those hypocentres with smaller data misfit. In this way all previous hypocentres guide the search, and the more promising regions of parameter space are preferentially sampled.

The NA procedure makes use of just two tuning parameters. It is possible to choose their values so that the behaviour of the algorithm is similar to that of a contracting irregular grid in 4-D. This is the feature of the algorithm that we exploit for hypocentre location. In experiments with different events and data sources, the NA approach is able to achieve comparable or better levels of data fit than a range of alternative methods; linearised least-squares, genetic algorithms, simulated annealing and a contracting grid scheme. Moreover, convergence was achieved with a substantially reduced number of travel-time/ slowness calculations compared with other nonlinear inversion techniques. Even when initial parameter bounds are very loose, the NA procedure produced robust convergence with acceptable levels of data fit.
\end{abstract}

Key words: Hypocentre, event location, neighbourhood algorithm.

\section{Introduction}

The location of seismic events in space and time is an important part of seismological practice with a wide range of applications, but has particularly significance in the context of monitoring a comprehensive test-ban treaty. A primary discriminant between man-made events and the majority of natural earthquakes is provided by the depth of the source. Drilling techniques for the emplacement of devices preclude a source depth of more than a few kilometres. Where the depth of an event can be established to be deeper than $15 \mathrm{~km}$, the event is very unlikely to be

${ }^{1}$ Research School of Earth Sciences, Institute of Advanced Studies, Australian National University, Canberra ACT 0200, Australia. E-mail: malcolm@rses.anu.edu.au, brian@rses.anu.edu.au 
man-made, even allowing for location errors. A significant role for location procedures in monitoring compliance with the treaty is therefore to act as a winnowing device to segregate the very large number of intermediate and deep-focus events occurring in subduction zones, especially around the Pacific Rim. For shallower events there is a need to obtain the best possible location by combining data from regional and more distant stations, and then to try to characterise the source using additional information.

One of the difficult tasks for a global monitoring system with a limited number of stations is the association of arrivals and waveform segments from a widely separated seismic stations with a single event. Across the network, it is quite likely that seismic phases from more than one event will be being recorded simultaneously. At least in the initial stages of location estimation, the procedures used should cope with the possibility that some phase readings may be misassociated, and not belong to the event of interest.

\section{Hypocentre Location}

In order to describe the hypocentre of a seismic event we have to specify the spatial location and the origin time of the event. These four pieces of information, the latitude, longitude, depth and the time of initiation of seismic energy have to be inferred indirectly. The primary information is the times of arrival of seismic phases at different stations; when seismic arrays are used these times can be supplemented with vector slowness information for phases, comprising the azimuth of the wavefront crossing the array and the horizontal slowness of the wavefront.

We can formulate the task of locating an event in the following terms: Given the arrival times [and slownesses] of seismic waves at a number of different receivers, deduce the best estimate of the origin time and spatial location of the hypocentre of the event (i.e., the point at which radiation is initiated). This task depends on us having a clear identification of the seismic phases whose arrival time [or slowness] is measured, and a good representation of the propagation processes associated with the measured passage times [or slownesses] for the different phases.

The task of phase association is not entirely straightforward but can be aided by the use of array information (see e.g., KvaERnA and Doornbos, 1986; Ringdal and KVAERNA, 1989) or by using multiple attribute analysis for three-component broadband records (CHRistoffersson et al., 1988; TONG and KenNeTt, 1995, 1996). In this work we will assume that the identity of each observed phase is known but allow for the possibility of using different classes of phases and types of information.

The approach used to determine an event location should not be limited by the representation of the propagation process. Ideally we would like to take account of the full three-dimensional structure of the earth, or at the very least allow for regional 
structure in the neighbourhood of the source. A practical first step is to determine a location with a spherically symmetric earth model, such as the iasp 91 model of KENNETT and ENGDAHL (1991) which is currently employed in the International Data Center for the CTBT, or ak135 (KENNETT et al., 1995) which has an improved representation for $S$ and core phases. Once an approximate location is established, region specific corrections can be applied or alternatively a more complex model representation used.

The specification of the location of the event requires the determination of four parameters

$t_{h}$ - the origin time of the seismic disturbance

$\mathbf{x}_{h}=\left(x_{h}, y_{h}, z_{h}\right)$ - the spatial coordinates of the hypocentre which together define the 4 -vector $\hat{\mathbf{h}}$ of hypocentral parameters.

We will assume that we have $N_{T}$ observations $t_{i}$ of the arrival times of identified phases at a set of seismic receivers with associated errors $\sigma_{i}$; these arrival times may be associated with different ray paths for a single wave type or different wave types. The time information may also be supplemented with $N_{S}$ observations of the vector slowness of arrivals (derived from seismic arrays).

For the $i$-th seismic phase we calculate the travel time $t_{r i}\left(\mathbf{x}_{s}\right)$ for passage between a source at $\mathbf{x}_{s}=\left(x_{s}, y_{s}, z_{s}\right)$ and the requisite receiver by ray tracing in an earth model. From these travel times we construct estimates $t_{c i}$ of the arrival times of the phases for an assumed origin time $t_{s}$ as

$$
t_{c i}\left(\mathbf{x}_{s}, t_{s}\right)=t_{s}+t_{r i}\left(\mathbf{x}_{s}\right)
$$

and these arrival times have to be compared with the observations $t_{i}$. We note the clear separation between the dependence of the estimated arrival time $t_{c i}$ on the spatial and temporal components of the estimated location.

In a similar way we construct estimates for the $j$-th vector slowness $\mathbf{s}_{c j}$ to be compared with the measured vector slownesses $\mathbf{s}_{j}$.

We now need to establish suitable measures of the extent to which the calculated arrival times and slowness match the observations so that we use these misfit measure to guide the inversion for the hypocentre $\hat{\mathbf{h}}$.

For arrival times the statistics of the residuals can often be well represented by a distribution suggested by JEFFREYs (1932) which consists of a Gaussian superimposed on a slowly varying pedestal function. Commonly, the narrower Gaussian provides a representation of the effect of picking errors and the broader background arises mostly from the differences between the real earth and the times predicted from a simplified model. The net effect is that the probability distribution has a substantially longer 'tail' than a Gaussian ( $L_{2}$ norm).

A simpler form with a similar property is to use $L_{p}$ measures of misfit with $p<2$. For many purposes, satisfactory results can be produced with $p=1.25$ which is tolerant to large outliers, even though this may not represent a close approximation to the actual probability distribution for the residuals (BILLINGS et al., 1994). In 
particular, when many different types of seismic phases are employed, the residual distributions can differ significantly between phases. An advantage of the $L_{p}$ measures with $p<1.5$ is that they are robust and much less affected by large outliers than the conventional squared residual measures.

We construct an $L_{p}$ measure for the misfit between observed and calculated arrival times as

$$
C_{T}=\left[\frac{1}{N_{T}} \sum_{i=1}^{N_{T}}\left|t_{i}-t_{c i}\right|^{p} / \sigma_{i}^{p}\right]^{1 / p},
$$

where $\sigma_{\mathrm{i}}$ is the estimate of the error in the $i$-th arrival time observation.

Array information can be included by the inclusion of an extra term in the misfit function (see e.g., BRATT and BACHE, 1988). For each vector slowness measurement $\mathbf{s}_{j}$, we construct corresponding theoretical slownesses $\mathbf{s}_{c j}$ for the current source location and calculate

$$
C_{S}=\left[\frac{1}{N_{S}} \sum_{j=1}^{N_{S}}\left|\mathbf{s}_{j}-\mathbf{s}_{c j}\right|^{p} / \sigma_{s j}^{p}\right]^{1 / p}
$$

where $N_{S}$ is the number of slowness measurements and $\sigma_{s j}$ is the error in the $j$-th slowness observation. Although the residual distribution for vector slowness is likely to be somewhat different from that for arrival times, we have achieved good results by using the same value of $p$ for both the time and slowness misfit terms $C_{T}$ and $C_{S}$.

We use the composite misfit function

$$
C=C_{T}+C_{S}
$$

as the measure of the quality of the location estimate.

\subsection{Nonlinear Inversion}

The conventional approach to event location is to use $L_{2}$ misfit representations in iterative inversion with linearisation around successive location estimates, which requires calculation of the derivatives of the times and slownesses with respect to the hypocentral parameters. The calculation of such derivatives limits the class of velocity models that can be conveniently employed.

However, with the advent of faster computers it is now feasible to calculate the travel times afresh for each postulated source location rather than rely on linearisation and so avoid any differentiation. This more flexible formulation allows us to use the more general representations of the misfit functions such as $L_{p}$ norms introduced above. For teleseismic arrivals, the tau-spline procedure of BULAND and ChAPMAN (1983) gives an efficient scheme for multi-phase travel time or slowness calculation for a given epicentral distance in a spherical model and has been adopted for the IASPEI91 travel-time tables (KENNETT and ENGDAHL, 1991). 
We have noted earlier the separation of the dependence of the arrival times for the seismic phases on the estimate of the origin time $t_{s}$ and the spatial location of the event $x_{s}$. This has been used as the basis of a number of different schemes in which a four-dimensional search procedure isolates the regions of best fitting hypocentres. For example, SAmbridge and KenNett (1986) used a directed spatial grid search with bracketing of the origin time and KENNETT (1992) introduced a contracting grid scheme which exploits the fact that the arrival times for different origin times do not require recalculation of travel times for a fixed source location. Such directed search procedures cannot guarantee that a global minimum for the misfit function will be found by the discrete search. However, the results obtained with different starting meshes are generally very consistent.

Alternative classes of nonlinear procedures based on direct calculation of travel times and different classes of optimisation algorithm have also been successfully used to find hypocentral estimates. Such methods include genetic algorithms (KenNETT and Sambridge, 1992; SAmbridge and Gallagher, 1993; Billings et al., 1994), simulated annealing (BILLINGS, 1994) and evolutionary programming (MINSTER et al., 1995). Even so, grid methods retain the attraction of simplicity of implementation and good performance even with poor network geometry.

In this paper we introduce the neighbourhood algorithm (NA) for location. The NA procedure exploits all previous information in the inversion procedure to focus in on those parts of parameter space for which the current misfit measure is least (SAMBRIDGE, 1999a). For the location problem the neighbourhood algorithm, as currently used, has approximately the character of a contracting irregular grid in four-dimensional hypocentre space. The procedure is very efficient and able to work with multiple local minima (SAMBridge, 1998).

\subsubsection{The use of a neighbourhood algorithm}

The neighbourhood algorithm is a derivative-free method for searching a multidimensional parameter space for models (hypocentres) of acceptable data fit. It is based on some simple geometrical concepts, involves two tunable parameters, and makes use of randomised, or stochastic, sampling of parameter space. We outline the approach below. Full details can be found in SAMBRIDGE (1999a).

The central idea behind the neighbourhood algorithm is the use of Voronoi cells to guide the search in parameter space. Voronoi cells are simply nearest neighbour regions, as defined by a suitable distance norm (Voronol, 1908; Delaunay, 1934). Here we use the $L_{2}$-norm. Figure 1 shows examples of Voronoi cells, defined about an irregular set of points in the plane. Each cell is a convex polygon about its defining point. Note that the sides of each cell are formed from the perpendicular bisectors between neighbouring pairs of points. Voronoi cells have many useful properties and have found applications in a number of fields (see OKABE et al., 1992, for a review). From Figure 1 we see that the size and shape of Voronoi cells automatically adapt to the density and distribution of the defining points. This is the property which is 

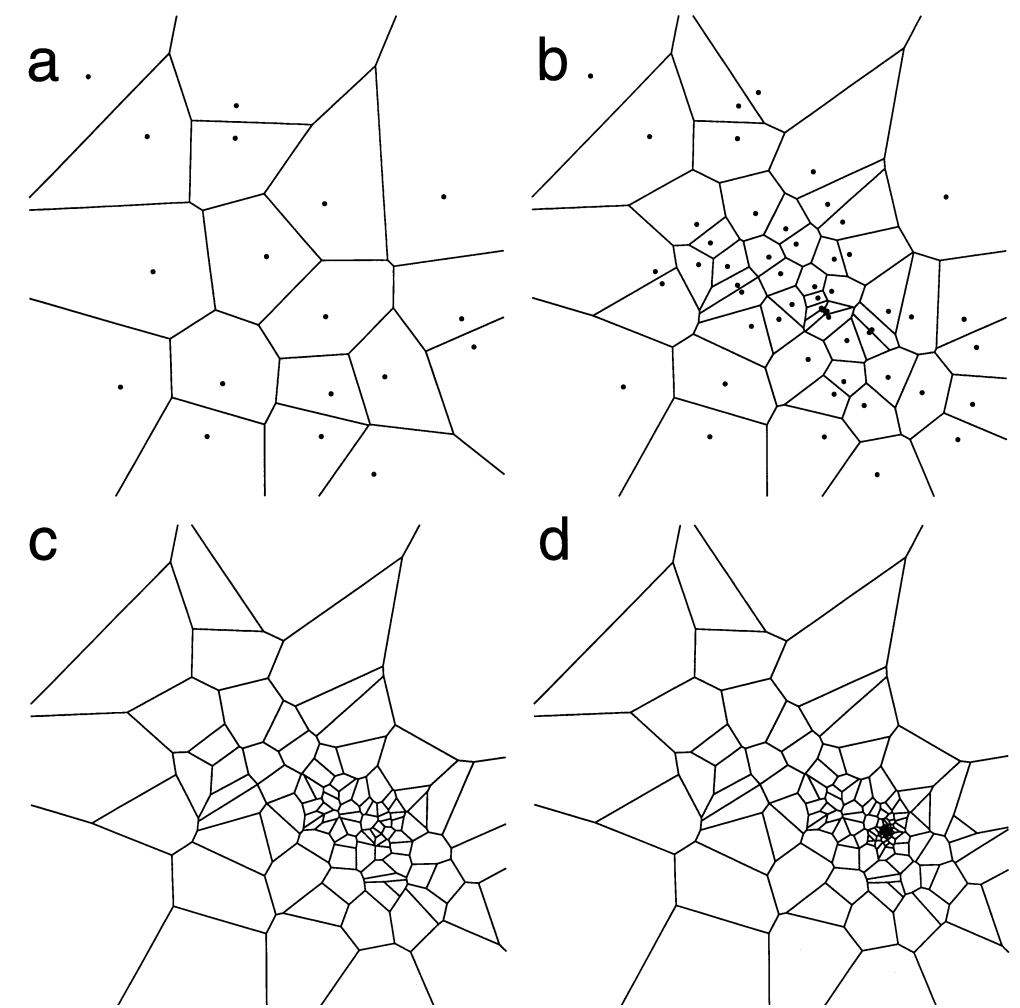

Figure 1

Voronoi cells produced by a neighbourhood algorithm after a) 1, b) 5, c) 10 and d) 20 iterations. At each iteration 9 samples are added into the cells about the 2 epicentres with the least data misfit.

exploited in the search algorithm. Figure 1 shows that the Voronoi cell defines a neighbourhood about each point.

The neighbourhood hypocentre location algorithm can be summarised as follows: First, an initial population of $n_{s}$ hypocentres is generated. Usually these follow a uniform random distribution within the parameter space bounds. At each iteration, the current $n_{r}$ hypocentres with lowest data misfit are determined and a new set of $n_{s}$ hypocentres is uniformly distributed inside their Voronoi cells, that is, $n_{s} / n_{r}$ in each cell. Subsequent iterations are repeated in a similar manner, with the $n_{r}$ best hypocentres and their Voronoi cells being updated at each iteration. The number of hypocentres per iteration $n_{s}$ and the number of Voronoi cells to be occupied $n_{r}$ are the two tuning parameters of the NA procedure. For each hypocentre tested, we calculate the associated arrival time and slowness information and use this to generate the appropriate misfit measure.

Note that the algorithm only uses the misfit measure to rank the goodness of fit of the hypocentres; the actual value of the misfit is not used directly. The use of a rank 
measure to drive the algorithm allows considerable freedom in controlling the way in which the data influence the NA search. For example, one may choose a single misfit measure combining arrival time and array measurements as in (4), with the relative weighting depending on the choice of assigned errors. Alternatively, one might separately rank hypocentral estimates according to the misfit for arrival time and array data; an overall rank could then be constructed from some combination of the two.

Figure 1, shows an example of the neighbourhood algorithm (used for epicentral location) at iterations $1,5,10$, and 20, with $n_{s}=9$ and $n_{r}=2$. Notice how the Voronoi cells adapt to the sampling and quickly focus in the southeastern part of the domain. The precise details of the sampling method are described in SAMBridge (1999a). It turns out that the bookkeeping calculations are surprisingly straightforward and may be handled very efficiently.

The behaviour of the search algorithm depends on the two control parameters, $n_{s}$ and $n_{r}$. As can be seen in Figure 1, for small values of $n_{r}$ it takes on the character of a contracting irregular grid, and can efficiently optimise a function. The power of the approach is due to the fact that at each iteration the sampling is driven by the size and distribution of the Voronoi cells, and these are controlled automatically by all previous samples.

\subsubsection{Location assessment}

In the absence of external information about the seismic source, the accuracy of the hypocentral location has to be estimated from the information available in the inversion. The implied precision of the estimates is therefore model-based and can be distinctly misleading as to the error in the location of the true hypocentre, e.g., through neglect of the influence of 3-D earth structure.

Commonly confidence intervals for the hypocentral location can be deduced by examining the distribution of

$$
\Gamma(h)=C(h)-C(\hat{h}),
$$

where $C$ is the misfit criterion and $\hat{h}$ is the best estimate of the location of the hypocentre.

The results from the neighbourhood algorithm inversion can be used a posteriori to build up an estimate of the probability distribution for the hypocentral estimates (SAMBRIDGE, 1999b). Bayesian measures of information (for example the posterior model covariance matrix, resolution matrix and marginal density distributions) may be useful in assessing the degree of constraint and trade-off between parameters. A fully nonlinear calculation of Bayesian measures involves the evaluation of various integrals of the posterior probability density distribution (PPD) over the hypocentral parameter space. SAMBRIDGE (1999b) describes a 'resampling' technique whereby estimates of these integrals may be obtained from the finite ensemble of hypocentres generated during the search stage. The accuracy of these measures depends in part on the size and distribution of the available ensemble. 
It is common for covariance and resolution matrices to be determined using local linearisation, for example, by approximating the contours of $\Gamma(h)$ by ellipsoids about the best fit hypocentre (for example, see BULAND, 1976). If the nonlinearity is severe, e.g., with a sparse network, then the linearised estimates are likely to be both inaccurate and biased. In such circumstances, we anticipate that the fully nonlinear approach would produce more accurate results. However, in either case knowledge of the statistics of all noise process in the data is required and this may be difficult in some cases.

\subsection{Illustrations of Event Location}

We illustrate the NA location scheme with two examples using different types of data. The first example is for a test event in the Marianas including arrival time and array information, and the second example is for $P$ arrivals from a nuclear blast at the E. Kazakh test site with known location. These examples have previously been used with other nonlinear procedures (KENNETT, 1992, 1996) and so provide a good base for comparative performance.

From a wide variety of tests we have chosen to use the NA procedure with an initial population of 9 random estimates spanning the search region and then 20 iterations in which 9 further estimates are produced at each stage. We have allowed resampling of up to 2 cells at each stage (i.e., $n_{s}=9$ and $n_{r}=2$ ). With these settings we have found that we achieve good convergence on a location estimate with a modest total of 189 sets of travel-time/slowness calculations.

\subsubsection{Arrival time and array slowness information}

The first example is based on an event in the Marianas. The arrival times have been supplemented with simulated array information, and so we use the extended definition of the misfit function.

The phase information used for this trial is presented in Table 1. Apart from two close stations, the data are from teleseismic distances with a limited range of azimuths. We have undertaken a number of different inversions for this data set using different styles of algorithms and different assumptions for constraints on the location.

For the first group of tests we have taken a region $2^{\circ}$ across in both latitude and longitude, with a depth interval of $60 \mathrm{~km}$ and a time interval of $40 \mathrm{~s}$ centred on the ISC location. We have then used a variety of different nonlinear algorithms with the IASPEI91 reference model. In each case we have used the same $L_{1}$-norm measure of misfit and we compare the results in Table 2. All these nonlinear methods give some shift from the ISC location derived using the Jeffreys-Bullen travel-time tables (without the array information). The smallest variations are obtained using the simulated annealing method [SA] (BILlingS, 1994), and a similar result with a shallower depth is also found from the use of the contracting grid scheme [CG] (KENNETT, 1992). By contrast the application of genetic algorithms [GA] (KENNETT 
Table 1

Marianas Test Event 20:17:49.4 14.5 N 146.9 E $33.00 \mathrm{~km}$

\begin{tabular}{|c|c|c|c|c|}
\hline Code & Phase & Hour Min Sec & Range [deg] & Back Azimuth \\
\hline GUA & Pn & 201824.20 & 2.05 & 242.4 \\
\hline GUMO & $\mathrm{Pn}$ & 201824.20 & 2.07 & 244.1 \\
\hline WB2 & $\mathrm{P}$ & 202452.20 & 36.35 & 200.0 \\
\hline BJI & $\mathrm{P}$ & 202457.00 & 36.92 & 319.4 \\
\hline PKI & $\mathrm{P}$ & 202743.60 & 58.23 & 293.6 \\
\hline KKN & $\mathrm{P}$ & 202744.50 & 58.34 & 293.8 \\
\hline DMN & $\mathrm{P}$ & 202745.80 & 58.50 & 293.6 \\
\hline $\mathrm{COL}$ & $\mathrm{P}$ & 202838.20 & 66.61 & 25.0 \\
\hline INK & $\mathrm{P}$ & 202917.00 & 72.82 & 22.5 \\
\hline YKA & $\mathrm{P}$ & 203004.40 & 81.20 & 27.7 \\
\hline SOD & $\mathrm{P}$ & 203035.00 & 87.64 & 340.4 \\
\hline $\mathrm{KJF}$ & $\mathrm{P}$ & 203041.00 & 88.98 & 337.5 \\
\hline SUF & $\mathrm{P}$ & 203048.00 & 90.37 & 336.6 \\
\hline NUR & $\mathrm{P}$ & 203056.00 & 92.21 & 335.2 \\
\hline HFS & $\mathrm{P}$ & 203117.30 & 96.64 & 338.4 \\
\hline Code & Phase & Azimuth & Range [deg] & Back Azimuth \\
\hline WB2 & $\mathrm{P}$ & 200.000 & 36.35 & 200.0 \\
\hline YKA & $\mathrm{P}$ & 104.250 & 81.20 & 27.7 \\
\hline Code & Phase & Slowness & Range [deg] & Back Azimuth \\
\hline WB2 & $\mathrm{P}$ & 0.080 & 36.35 & 200.0 \\
\hline YKA & $\mathrm{P}$ & 0.050 & 81.20 & 27.7 \\
\hline
\end{tabular}

Table 2

Comparison of location estimates

\begin{tabular}{cccccc}
\hline Method & Lat. & Long. & Depth $[\mathrm{km}]$ & Time & $\mathrm{L}_{1}$ Misfit \\
\hline ISC & $14.50 \mathrm{~N}$ & $146.90 \mathrm{E}$ & 33.00 & 49.40 & 1.380 \\
SA & $14.51 \mathrm{~N}$ & $146.92 \mathrm{E}$ & 23.10 & 49.44 & 0.755 \\
CG & $14.52 \mathrm{~N}$ & $146.90 \mathrm{E}$ & 22.80 & 49.40 & 0.748 \\
GA 1 & $14.49 \mathrm{~N}$ & $146.67 \mathrm{E}$ & 49.97 & 53.35 & 0.732 \\
GA 2 & $14.50 \mathrm{~N}$ & $146.72 \mathrm{E}$ & 44.10 & 52.70 & 0.720 \\
NA & $14.50 \mathrm{~N}$ & $146.78 \mathrm{E}$ & 41.64 & 52.23 & 0.711 \\
\hline
\end{tabular}

and SAMBridge, 1992) produce a better fit with a deeper source; two different GA results are displayed based on the use of different seed for the random processes. For each of these GA inversions 1320 sets of travel-time evaluations were undertaken to arrive at the location estimate. The neighbourhood algorithm produces a result with the smallest misfit of any of the methods. The NA method employs only 189 traveltime and slowness evaluations which is less than a quarter of that for any of the other techniques. 
The NA procedure does not depend on having a good initial estimate of the location. We illustrate this for the Marianas event event by taking a starting model displaced by more than $100 \mathrm{~km}$ from the likely location and with a significant time offset. We employ a grid with a $\pm 2^{\circ}$ range in latitude and longitude, $\pm 40 \mathrm{~km}$ range in depth and $\pm 30 \mathrm{~s}$ in origin time about $13.52 \mathrm{~N}, 147.5 \mathrm{E}, 39.0 \mathrm{~km}, 47.9 \mathrm{~s}$ for which the $L_{1}$ misfit is 1.916 . We show in Figure 2 the progress of the NA scheme with an $L_{1}$ measure of misfit towards the best fitting location by means of projections onto the spatial axes and a time bar. The display covers almost the entire allowed ranges on the parameters.

The neighbourhood algorithm initially explores much of the allowed domain but quite quickly shifts attention to a zone with improved misfit. The convergence towards a cluster of well-fitting location estimates is rapid even though the starting point is well displaced from the best estimate of the location. Each of the samples tested in an individual iteration is indicated by the same symbol. As the iteration number increases the number of sides is also increased and then solid symbols are introduced for the last few iterations. The best fit location is very close to that reached with the domain centred at the ISC solution [14.484 N, $146.761 \mathrm{E}$, $43.156 \mathrm{~km}, 52.30 \mathrm{~s}$ ] with an $L_{1}$ misfit of 0.716 .

The progress of the NA procedure for this case is illustrated in Figure 3. The initial model estimate used as the centre of the bounds on the parameter distribution provides a moderately good fit. It requires several iterations of the exploration of the model space to find a better model. Exploration continues and from time to time a model is found which has lower misfit than all its predecessors. As the 20th iteration is approached, all models are tightly clustered around the best fit (as can also be seen in Fig. 2).

As a final test for this same data set we have undertaken an inversion with a very broad set of initial bounds $\left[ \pm 5^{\circ}\right.$ in latitude and longitude, $\pm 60 \mathrm{~km}$ in depth and $\pm 60 \mathrm{~s}$ in origin time] about the same starting point as in the previous example. If we run the NA procedure for 20 iterations as in the previous example we obtain a very satisfactory location given the weak imposed constraints: [14.441 N, $146.625 \mathrm{E}$, $59.03 \mathrm{~km}, 52.30 \mathrm{~s}$ ] with a misfit of 0.780 . This solution is not far from that found with much smaller bounds but has higher misfit. This shows that with the control parameters used in this case, it is possible for the NA search to get distracted by local minima and therefore not reach the best possible solution.

With a low cost approach such as the neighbourhood algorithm it is possible to construct solutions from a number of different starting points and accept that which gives the best fit. Thus with the same coarse grid around the centre point $[14.0 \mathrm{~N}$, $146.0 \mathrm{E}, 50.0 \mathrm{~km}, 58.0 \mathrm{~s}$ ] we reach an $L_{1}$ misfit of 0.712 at [14.496 N, $146.734 \mathrm{E}$, $45.189 \mathrm{~km}, 52.64 \mathrm{~s}]$ in 189 sets of travel-time calculation.

Once a reasonable solution has been found it is also effective to restart the NA search with a smaller zone in order to refine the hypocentre. In our experience the neighbourhood algorithm is the most robust method for assessing likely locations when the bounds on the allowed solution are very large, as is likely to be the case 


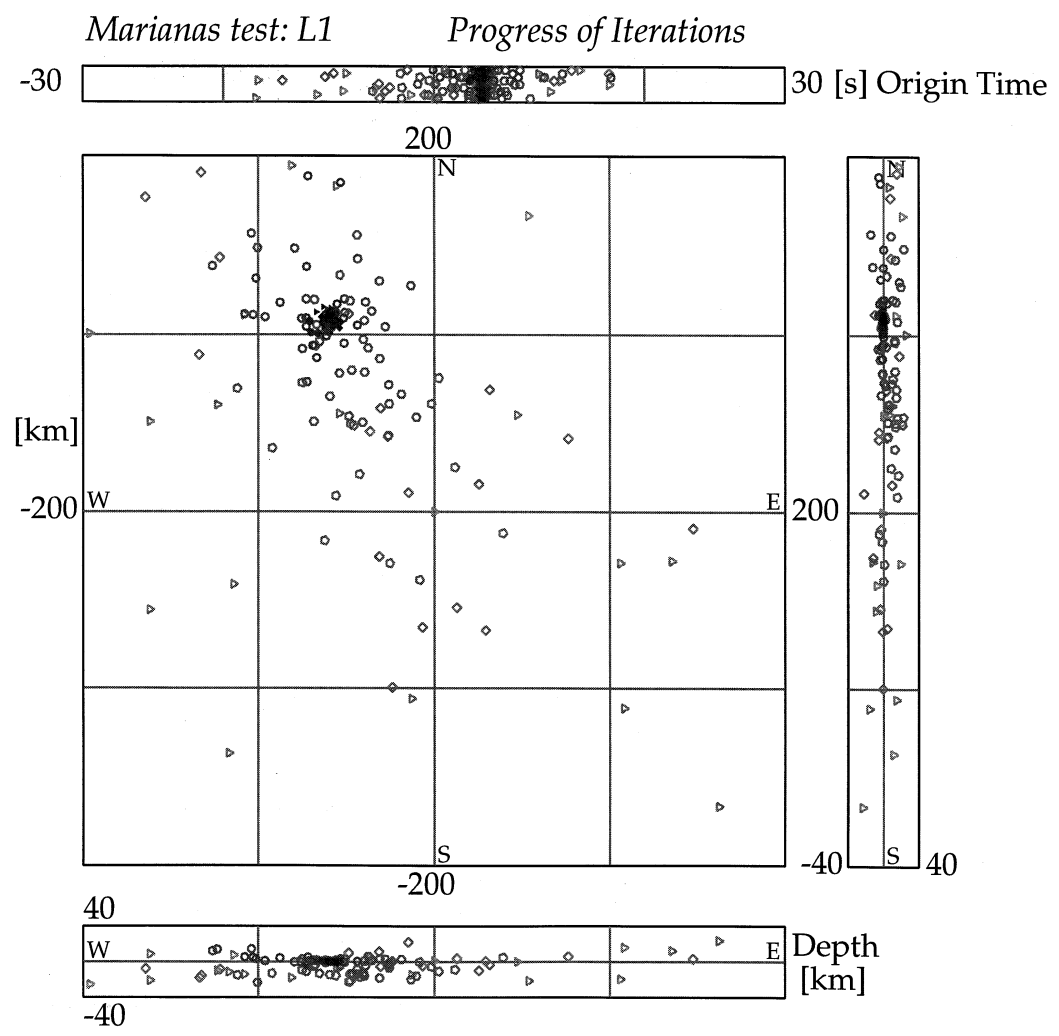

Figure 2

Representation of the progress of the NA technique towards convergence on a location estimate via projections onto the different spatial planes. The centre is the reference point about which the parameter bounds were specified. The group of models generated at each iteration are indicated by the same style of polygons. The number of sides increases as the iterations proceed, but for clarity solid symbols are introduced for the last few iterations.

with the sparse global IMS network operating under the Comprehensive NuclearTest-Ban Treaty.

\subsubsection{East Kazakh event}

In the second example we consider a nuclear test at the Semipalatinsk test site for which precise coordinates are available from Soviet sources (BOCHAROV et al., 1989). We have chosen one of the smaller events and used the set of arrival times reported by the Bulletin of the International Seismological Centre. The station distribution relative to the event is illustrated in Figure 4, and displays a high concentration of stations in Europe with a scatter across the rest of the globe and a broad open azimuth sector towards the Pacific. There are no close stations so that the resolution in depth is expected to be rather poor. 


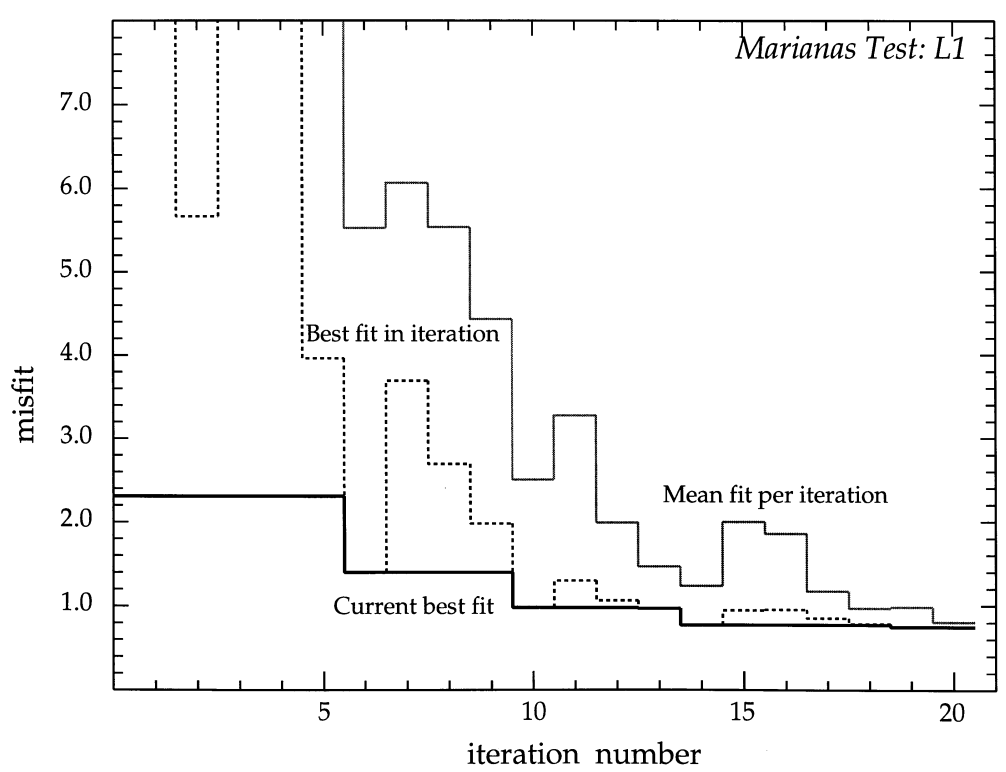

Figure 3

The progress of convergence in the NA procedure for the Marianas test. The best fitting model per iteration, the mean misfit across all the models in each iteration and the current best fit are shown. All converge over the 20 iterations of the neighbourhood algorithm.

We illustrate the progress of the hypocentral inversion in Figure 5 with an $L_{1.25}$ measure of misfit using a range of $\pm 1^{\circ}$ in latitude and longitude, $\pm 10 \mathrm{~km}$ in depth and $\pm 10 \mathrm{~s}$ in origin time, centred at the Soviet location. Each of the symbols in Figure 5 represents one of the location estimates which has been assessed in the progress of the NA inversion. The symbols are coded in greytone by the level of misfit with darker tones indicating better fit. The display in Figure 5 shows only a portion of the original search region and therefore displays the nature of the misfit function in the neighbourhood of the best fitting model. The lightest symbols have a misfit approximately twice that of the best. The immediate neighbourhood of the best locations indicates relatively slow variation but away from this region the misfit in arrival times grows quite rapidly. As indicated above the sampling of the misfit function in the course of the NA inversion can be put to use in a retrospective assessment of the probability distribution for the hypocentre parameters.

The Soviet location (at the centre of the main grid) is one of the models tested in the NA process but does not provide the best fit because of the influence of both 3-D heterogeneity in the earth and the geometry of the available network. In all the different inversions we have tried, the best fitting location is displaced about $6 \mathrm{~km}$ to the east and $1 \mathrm{~km}$ to the south of the true location. With the IASPEI91 travel times the origin time lies within $0.6 \mathrm{~s}$ of the Soviet information. Because of the limited 


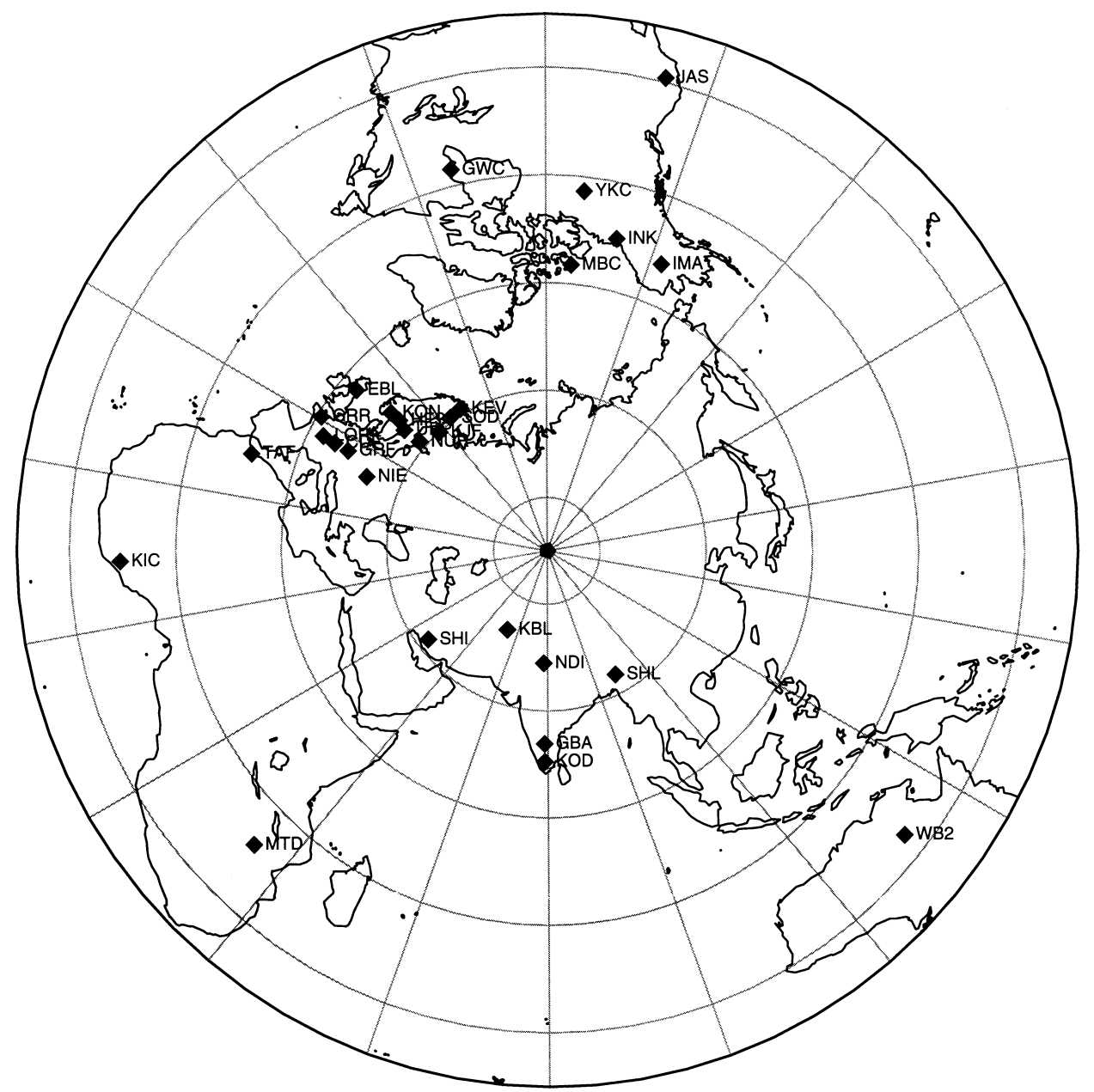

Figure 4

The distribution of stations used in the hypocentral estimates for the East Kazakh event. Polar projection centred on the announced hypocentre.

depth constraints the depth estimate depends more strongly on the range of allowed depths. With an allowed depth range from $0-50 \mathrm{~km}$ we find a depth of $7 \mathrm{~km}$ and tighter limits on the depth sampling produce shallower solutions with a misfit that decreases slowly with decreasing depth. The best fit is achieved for a constrained solution at the Soviet depth of $0.139 \mathrm{~km}$ but with only a very small shift in horizontal position (about $0.25 \mathrm{~km}$ ).

As discussed in section 2.1, it is possible to make use of the ensemble of hypocentral estimates generated in the NA search to generate an a posteriori probability distribution for the hypocentral parameters. 


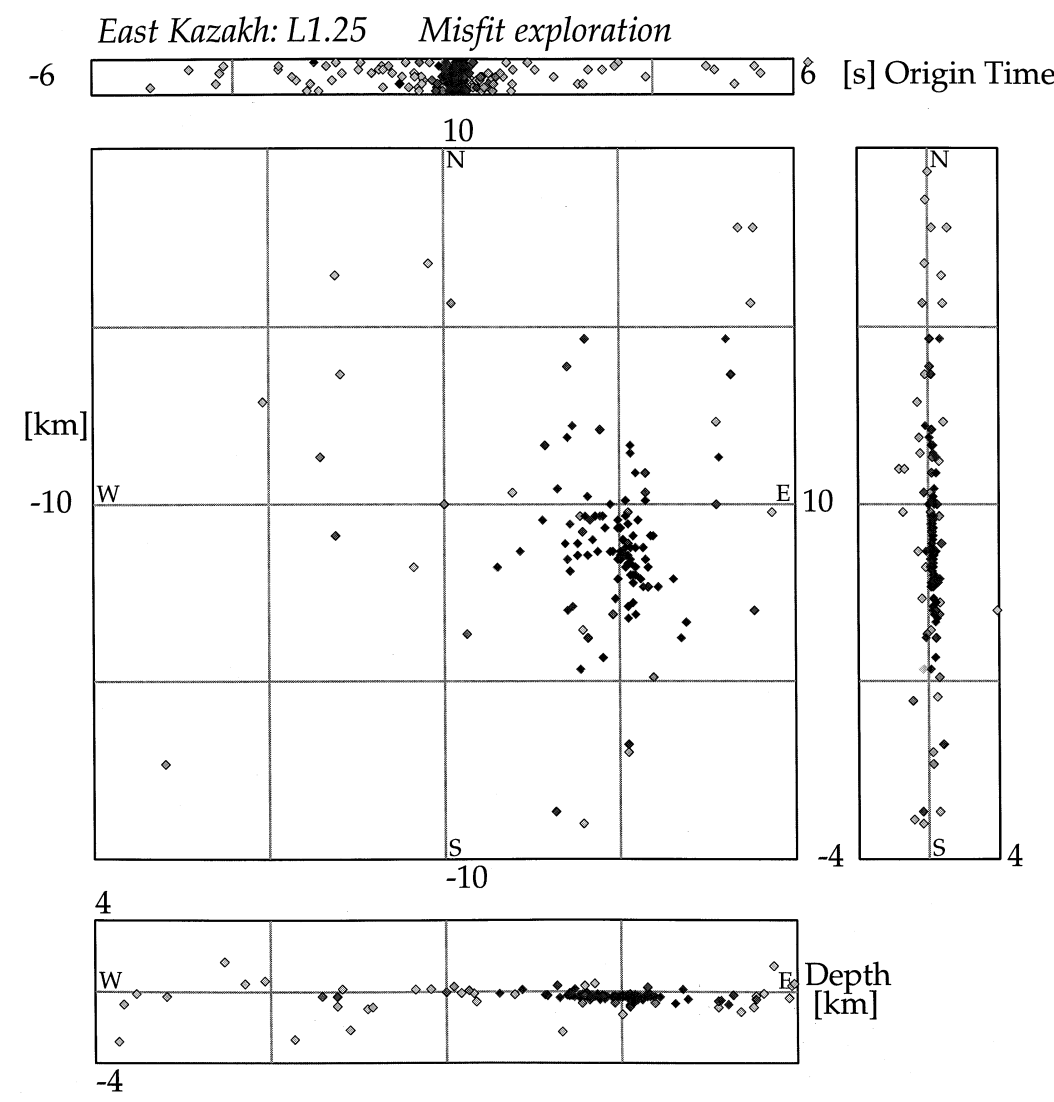

Figure 5

The central portion of the parameter space showing the sampling of model misfit in the NA procedure. The darker symbols indicate better fit and their distribution indicates the reliability of the estimated hypocentre. The environs of the best fitting model are well explored and this information is available for assessment of the probability distribution of the hypocentral parameters.

The distribution of misfit in hypocentral space can also be used directly as an indication of the reliability of the postulated hypocentre. If we can prescribe a satisfactory level of fit, then the 'cloud' of hypocentres whose misfit lies below this level outline a nonlinear error domain for the solution. The process can be illustrated with Figure 5 where the cluster of the darkest symbols (indicating least misfit) occupy a zone about $3 \mathrm{~km} \mathrm{E} / \mathrm{S}$ by $5 \mathrm{~km} \mathrm{~N} / \mathrm{S}$.

The quality of the location estimates we have achieved using the IASPEI91 travel times is very good when we allow for the limited data set and the influence of lateral heterogeneity. Inclusion of site specific corrections would readily shift the solution to the 'ground truth'. 


\subsubsection{Phase rejection during location}

For any global network, a significant aspect of the location of seismic events is the separation of seismic phase information for a particular time period into sets of information associated with separate events. In an initial scan it may not be apparent whether certain observations should be associated with others in the definition of an event.

KENNETT (1996) has shown how a contracting grid procedure with a robust measure of misfit such as an $L_{1}$ or $L_{1.25}$ norm can be used to recognise observations which do not match with the main group of phase readings, so that these can be removed from further consideration. The approach is to undertake a preliminary inversion over a wide area (e.g., $20^{\circ}$ across and $200 \mathrm{~km}$ deep) with a coarse initial grid and look for a location estimate with the least misfit even though the variation in the misfit may be small because of the major outliers. This approximate location is then used to identify those observations which are regarded as having too large residuals to be reliably associated with the event. The data set is then reduced to include only the consistent information and then a finer grid search, over a smaller region, is used to improve the location estimate.

This style of procedure is readily adapted to use with the neighbourhood algorithm. Once again the initial model scan is over a broad region and the algorithm is run for a limited number of iterations and then the best fitting location estimate is used to screen the observations. We recall that we depend only on the ranking of the misfit of the different location estimates and the algorithm is not affected by a large bias from the discordant observations. Once the data set is reduced the neighbourhood algorithm is used over a smaller zone such as those employed above to improve the estimate of the event location.

\section{Discussion}

The neighbourhood algorithm is able to achieve good quality locations with far fewer travel-time evaluations than any previous nonlinear location procedure we have tried. In the examples we have shown we have been able to get good results with a total of 189 travel-time computations. In contrast the contracting grid scheme of KENNETT (1992) requires at least 500 travel-time computations to achieve comparable levels of misfit. Note that trial bounds can be specified independently on each parameter so that it is possible to impose strong constraints on the depth of an event if required.

The particular advantage of the neighbourhood algorithm approach is that the computational effort is concentrated on those portions of hypocentral space where the misfit is least, and further the scheme can follow multiple minima (SAMBridge, 
1998) and therefore provide information on alternative location possibilities. When combined with the retrospective assessment of the probability distribution of the location parameters, the scheme provides both an effective location estimation and assessment procedure which can be used with any class of velocity model for which ray-tracing can be undertaken including full 3-D structure.

\section{REFERENCES}

Billings, S. (1994), Simulated Annealing for Earthquake Location, Geophys. J. Int. 118, 680-692.

Billings, S., Kennett, B. L. N., and SAmbridge, M. S. (1994), Hypocentre Location: Genetic Algorithms Incorporating Problem-Specific Information, Geophys. J. Int. 118, 693-706.

Bocharov, V. S., Zelentsev, A., and Mikhailov, V. N. (1989), Characteristics of 96 Underground Nuclear Explosions at the Semipalatinsk Test Site (in Russian), Atomnaya Energiya 67 (3).

Bratt, S. R., and BACHE, T. C. (1988), Locating Events with a Sparse Network of Regional Arrays, Bull. Seismol. Soc. Am. 78, 780-798.

Buland, R. (1976), The Mechanics of Locating Earthquakes, Bull. Seismol. Soc. Am. 66, 173-187.

Buland and Chapman (1983) The Computation of Seismic Travel Times, Bull. Seismol. Soc. Am., 73, $1271-1302$.

Christoffersson, A. E., Husebye, E. S., and Ingate, S. F. (1988), Wavefield Decomposition Using ML Probabilities in Modelling Single Site 3-component Records, Geophys. J. Int. 93, 197-213.

Delaunay, B. N. (1934), Sur la sphere vide, Bull. Acad. Science USSR VII: Class. Sci. Math., 793-800. Jefrreys, H. (1932), An Alternative to the Rejection of Observations, Proc. R. Soc. Lond. 137A, 78-87.

KennetT, B. L. N. (1992), Locating Oceanic Earthquakes - The Influence of Regional Models and Location Criteria, Geophys. J. Int. 108, 945-854.

KenNetT, B. L. N. Event location and source characterisation. In Monitoring a Comprehensive Test-BanTreaty (eds. Husebye, E. S., and Dainty, A.) (Kluwer, Dordrecht 1996) pp. 501-520.

Kennett, B. L. N., and ENGDAhl, E. R. (1991), Travel Times for Global Earthquake Location and Phase Identification, Geophys. J. Int. 105, 429-465.

Kennett, B. L. N., and Sambridge, M. S. (1992), Earthquake Location: Genetic Algorithms for Teleseisms, Phys. Earth. Planet. Int. 75, 103-110.

Kennett, B. L. N., Engdahl, E. R., and Buland, R. (1995), Constraints on Seismic Velocities in the Earth from Travel Times, Geophys. J. Int. 122, 108-124.

Kvaerna, T., and Doornbös, D. J. (1986), An integrated approach to slowness analysis with arrays and three-component stations, Semiannual Technical Summary, 1 October 1985-31 March 1986, NORSAR Sci. Report No. 2-85/86, Kjeller, Norway.

Minster, J. -B. H., Williams, N. P., Masters, T. G., Gilbert, J. F., and HaAse, J. S., Application of evolutionary programming to earthquake hypocenter determination. In Proc. 4th Annual. Conf. of Evol. Prog. (eds. McDonnell, J. R., Reynolds, R. C., and Fogel, D. B.) (MIT Press, Cambridge 1995) pp. 3-17.

Okabe, A., Boots, B., and Suginara, K., Spatial Tessellations Concepts and Applications of Voronoi Diagrams (John Wiley \& Sons, New York 1992).

Ringdal, F., and Kvaerna, T. (1989), A multi-channel Processing Approach to Real-time Network Detection, Phase Association and Threshold Monitoring, Bull. Seismol. Soc. Am. 79, 1927-1940.

SAmbridge, M. S. (1998), Exploring Multidimensional Landscapes without a Map, Inverse Problems 14, $427-440$.

SAmbridge, M. S. (1999a), Geophysical Inversion with a Neighbourhood Algorithm - I. Searching a Parameter Space, Geophys. J. Int. 138, 479-494.

SAmbridge, M. S. (1999b), Geophysical Inversion with a Neighbourhood Algorithm - II. Appraising the Ensemble, Geophys. J. Int. 138, 727-746.

Sambridge, M. S., and Kennett, B. L. N. (1986), A Novel Method of Hypocentre Location, Geophys. J. R. Astr. Soc. 87, 313-331. 
Sambridge, M. S., and Gallagher, K. (1993), Earthquake Hypocenter Location Using Genetic Algorithms, Bull. Seismol. Soc. Am. 83, 1467-1491.

Tong, C., and KennetT, B. L. N. (1995), Towards the Identification of Later Seismic Phases, Geophys. J. Int. $123,948-958$.

Tong, C., and Kennett, B. L. N. (1996), Automatic Seismic Event Recognition and Later Phase Identification for Broad-band Seismograms, Bull. Seismol. Soc. Am. 86, 1896-1909.

Voronoi, M. G. (1908), Nouvelles applications des paramètres continus à la théorie des formes quadratiques, J. reine angew. Math. 134, 198-287.

(Received June 30, 1999, revised October 6, 1999, accepted February 9, 2000)

\section{(1) To access this journal online: \\ (20) http://www.birkhauser.ch}

